\title{
Accurate Antenna Pattern Modeling for Phased Array Antennas in SAR Applications-Demonstration on TerraSAR-X
}

\author{
Markus Bachmann, Marco Schwerdt, and Benjamin Bräutigam \\ German Aerospace Center (DLR), Oberpfaffenhofen, 82234 Weßling, Germany \\ Correspondence should be addressed to Markus Bachmann, markus.bachmann@dlr.de
}

Received 22 October 2008; Revised 10 February 2009; Accepted 13 March 2009

Recommended by Stefano Selleri

\begin{abstract}
The high flexibility and tight accuracy requirements of today's spaceborne synthetic aperture radar (SAR) systems require innovative technologies to calibrate and process the SAR images. To perform accurate pattern correction during SAR processing, an Antenna Model is used to derive the multitude of different antenna beams generated by active antenna steering. The application of such an Antenna Model could be successfully demonstrated for the TerraSAR-X mission, launched in 2007. The methodology and the results of the inorbit verification with an achieved accuracy of better than $\pm 0.2 \mathrm{~dB}$ is reviewed in this paper in detail showing its outstanding accuracy.
\end{abstract}

Copyright (C) 2009 Markus Bachmann et al. This is an open access article distributed under the Creative Commons Attribution License, which permits unrestricted use, distribution, and reproduction in any medium, provided the original work is properly cited.

\section{Motivation}

The accurate knowledge of the antenna patterns of a synthetic aperture radar (SAR) is of main importance for precise SAR image processing. The antenna patterns are required to correct the antenna characteristics visible in the image, as shown in Figure 1. On the left, the uncorrected image is shown with a high brightness in the centre and a decreasing illumination to the borders of the image in range direction. This is caused by the antenna pattern spanning over range. The right image presents the same acquisition after antenna pattern correction, the characteristics of the antenna are eliminated.

Early SAR systems like ERS1/2 or XSAR/SRTM with a low number of possible antenna beams used inorbit antenna pattern measurements for correction. The Envisat/ASAR instrument, for example, acquires SAR images with only eight different antenna beams [1]. Although already at ASAR an Antenna Model was implemented, the more accurate antenna patterns were obtained from inorbit measurements. Images over homogeneously distributed targets were used to determine the reference patterns for each individual beam.

In contrast to these systems, actual SAR satellites enable a very high number of different acquisition modes like stripmap, ScanSAR or other wide swath and high resolution modes. Thus, a multitude of different antenna beams and hence antenna patterns are needed for consistent calibration of the modes to each other and within the SAR image itself.

Enabling object detection and classification novel SAR systems have also very tight accuracy requirements. They produce images with high resolution in the meter-range and accurate measures of the backscatter down to a few tenth of $\mathrm{dB}$.

A further important point for satellite calibration is the duration of the calibration process, the commissioning phase of the satellite in space. Current SAR systems are no longer experimental systems designed for scientific experiments having uncritical schedule constraints. In fact they shall, to a great extend, be used for commercial applications. Driven by paying customers, the system has to be available as early as possible or at least at an exactly determined date. Hence, a short duration of the commissioning of the satellite is evident.

Reviewing these points, innovative methods are necessary to calibrate complex SAR systems. In this context, the most important key element is the Antenna Model approach described below. It derives the antenna patterns from mathematical models in combination with onground measurements characterising parts of the array antenna. With this approach it becomes possible to calibrate a high 


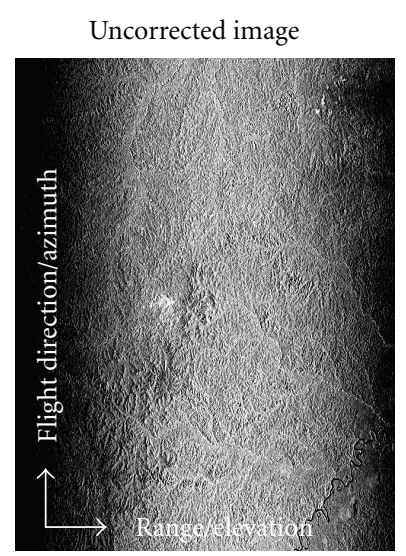

(a)
After antenna pattern correction

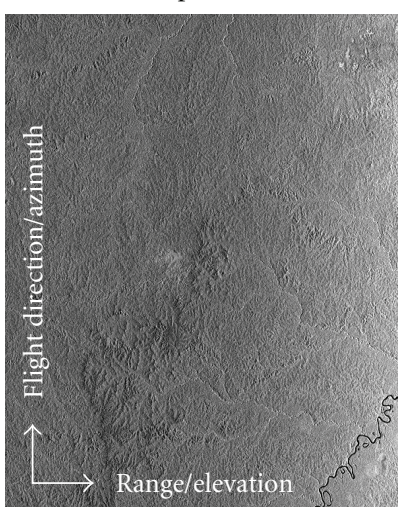

(b)
FIGURE 1: SAR image before and after pattern correction.

number of used antenna beams not only with high accuracy but also very time and cost effective. This is demonstrated in example of the TerraSAR-X system.

\section{Introduction on the TerraSAR-X System}

The TerraSAR-X satellite [2], launched in June 2007, is a versatile X-Band SAR satellite built in a Public Private Partnership (PPP) between the German Aerospace Center (DLR) and Astrium GmbH. The main payload of TerraSAR$\mathrm{X}$ is an SAR instrument to acquire high quality radar images of the Earth's surface.

The SAR instrument comprises an active phased array antenna which allows flexible beam forming. The antenna with its $4.8 \mathrm{~m}$ length and $0.7 \mathrm{~m}$ width consists of 384 subarrays composed by two slotted wave-guides, one for each polarisation (horizontal and vertical). These are arranged in 12 panels in azimuth direction (columns) each composed of 32 subarrays (rows) [3]. The nominal antenna pointing in elevation is $33.8^{\circ}$ away from nadir. Right and left looking acquisition is realised by satellite roll manoeuvres. Each individual subarray is driven by a Transmit/Receive Module (TRM) adjustable in amplitude and phase by applying complex excitation coefficients. This enables beam steering and adaptive beam forming in both azimuth and elevation direction. More than 12000 different beams can be commanded for the multitude of standard acquisition modes possible on TerraSAR-X. These are the nominal Stripmap, ScanSAR or Spotlight modes as well as several experimental modes like quad-polarisation mode, wide band operation, or along-track interferometry.

\section{Antenna Model Approach}

The Antenna Model is used to compute this huge amount of antenna patterns needed by the processing system to correct the impact of the antenna characteristics on the radar images. In elevation, the antenna patterns are then used for direct image correction over range. The azimuth pattern is represented in the Doppler spectrum and hence needed for correct Doppler estimation.

The optimisation of the beam excitation coefficients of the antenna array is a second important task that can be performed with the Antenna Model. These excitation coefficients are complex values which are applied on the TRMs in order to steer the beam in the desired direction as well as to create an antenna pattern with an optimised gain, a desired pattern slope or suppressed side-lobes. With the optimisation process, an optimum set of excitation coefficients and hence an optimum performance for the full performance beams in terms of Noise Equivalent Sigma Zero (NESZ) and Total Ambiguity Ratio (TAR) are achieved.

Also, in case of contingences like TRM degradation or failures during the operational phase resulting in a degradation of the antenna patterns, the antenna excitation coefficients can be re-optimized to ensure the high performance.

The development and the establishment of an Antenna Model approach were driven by three main requirements.

(i) The great number of more than 12000 different beams to be calibrated.

(ii) The tight accuracy requirement of an overall radiometric accuracy of better than $1.0 \mathrm{~dB}$ ( 1 sigma). This value was derived from the radiometric error budget calculated prior to the development of TerraSAR-X. All radiometric errors affecting the SAR acquisition were considered in this error budget. The main contributions are the accuracy of the internal calibration, the error of the antenna model, the mechanical and electrical antenna pattern variation, processing errors, the accuracy and stability of the measurement targets and atmospheric variations. In this budget, the requirement for the accuracy of the Antenna Model is to be better than $\pm 0.2 \mathrm{~dB}$ (peak-to-peak) for reproducing the pattern shape and predicting the gain offset between different beams. Another important reason for this number is that a deviation of more than $0.2 \mathrm{~dB}$ can visibly be recognized in overlayed or adjacent SAR images.

(iii) The short duration of the commissioning phase of less than six months.

To ensure these requirements, several steps were realized.

(i) As much effort as possible was moved from inorbit tasks to onground duties. This includes the accurate measurement of the embedded subarray patterns as well as the validation of the model onground before launch. The task was successfully performed by Astrium and DLR, and is described in more detail in Section 5.

(ii) Different inorbit calibration techniques for Antenna Model verification were used. Therefore, inorbit verification was performed during the commissioning phase in the first months after launch. 
The verification was divided into three main tasks:

(a) measurements across the rainforest to verify the elevation pattern shape,

(b) the use of ground receivers to verify the azimuth pattern and,

(c) ScanSAR measurements over rainforest and over ground receivers to verify the prediction of the gain offset between the beams. This task is described in detail in Section 4.

(iii) To ensure a short commissioning phase, the verified Antenna Model supported the absolute radiometric calibration which is the determination of the absolute calibration factor. With a verified Antenna Model, the absolute calibration factor of only one beam would have to be determined, which minimizes the effort for the absolute radiometric calibration significantly. A description of the absolute radiometric calibration would exceed the content of this paper and the interested reader is referred to $[4,5]$.

The described approach is summarized in Figure 2. Starting from the Antenna Model design, the model is validated onground first. After launch, the verification of the model is performed over distributed targets like rainforest and point targets like ground receivers. Hereby the actual state of the instrument and the TRMs is monitored simultaneously by applying the Internal Calibration facility and the so-called PN gating method in the instrument.

The Internal Calibration measures the actual state of the transmit and receive path within the radar instrument, which afterwards can be corrected during image processing. Therefore, it is performed at every beginning and end of an acquisition.

The PN-Gating method [6] is a novel approach to monitor the actual state of each individual TRM. It was demonstrated on TerraSAR-X for the first time inorbit. In contrast to the module stepping approach applied on Envisat/ASAR where each TRM was measured sequentially, the TRMs are characterised during quasinominal operation that means with all TRMs enabled. Hence, the TRMs are driven under most realistic conditions while they are characterised. This is realised by applying orthogonal PN- or Walsh-codes to the TRMs. The code consists of up to 512 code chips with one individual code at each TRM enabling the extraction of each individual TRM. The PN-Gating method is applied regularly to detect changes, drifts or failing TRMs. If the changes exceed a given limit, the antenna patterns have to be recalculated or even re-optimised.

By this Antenna Model sufficiently validated onground and verified by a limited number of a few selected beams really measured inflight, the thousands of reference patterns can be accurately derived.

\section{Antenna Model Design}

The Antenna Model itself mathematically calculates radiation patterns by the superposition of four inputs: (i) radiation patterns measured onground from the elements of the antenna, so-called embedded subarray patterns,

(ii) beam excitation coefficients (amplitude and phase) of each individual transmit/receiver module (TRM),

(iii) exact geometrical dimensions of the array antenna including the distances between the subarrays

(iv) actual state of the SAR instrument like drifting and/or failed individual TRMs.

For active phased array antennas, the radiated pattern $F_{\text {Beam }}$ is calculated by $[3,7]$ :

$$
\begin{array}{r}
\vec{F}_{\text {Beam }}(\varepsilon, \alpha)=\sum_{m=0}^{M-1} \sum_{n=0}^{N-1}\left(\vec{C}_{\mathrm{SA}, m n}(\varepsilon, \alpha) \cdot a_{m n} \cdot E_{\mathrm{SA}, m n}\right. \\
\cdot e^{j k \sin \varepsilon \cos \alpha(-(N-1) / 2+n) \Delta y} \\
\left.\cdot e^{j k \cos \varepsilon \sin \alpha(-(M-1) / 2+m) \Delta x}\right)
\end{array}
$$

with the desired elevation and azimuth angles $\varepsilon$ and $\alpha$, the amount of subarrays in $N$ rows and $M$ columns, the intersubarray distances $\Delta x$ (columns) and $\Delta y$ (rows). The wave number $k$ includes the centre frequency $9.65 \mathrm{GHz}$ of the system by the relation of $k=2 \pi / \lambda$.

The embedded subarray patterns $C_{S A}$ comprise the real measured radiation characteristics of the individual subarray elements. Therefore, the subarrays are embedded into the whole antenna and have to be given for each row, column, elevation and azimuth angle. The embedded pattern of one subarray mounted in the array antenna describes the radiation characteristic of this subarray. In this way, mutual coupling effects are included by the measurements. The antenna has a quite broad frequency bandwidth of $300 \mathrm{MHz}$, so that mismatch and gain are smooth over the frequency bandwidth. Therefore, only the embedded patterns at centre frequency are incorporated in the Antenna Model.

As input for the antenna model, all embedded subarrays of one panel were measured. The patterns of whole panels are very similar to each other, even for the ones at the edges. The measurement results showed that it is possible to use only the embedded patterns of one panel and substitute the others using their measured amplitude and phase offsets. These embedded patterns have to be known with a very high accuracy, as they are one main input of the model.

The commanded complex excitation coefficients are given by $a$. In case of TerraSAR-X, these coefficients are provided in form of amplitude and phase values row and column-wise. For each commandable beam, one set of values is put in a common table. This table is available onboard the satellite and can be updated if necessary, for example, in case of contingencies, as the onboard computer uses the table for each acquisition.

Finally, the error matrix $E_{\mathrm{SA}}$ describes drifting or failed antenna elements which are determined via the PN-Gating method using orthogonal code sequences applied to the TRMs of the instrument as described in Section 3.

To obtain the complete two-way antenna patterns, the equation is evaluated for transmit and receive individually. 


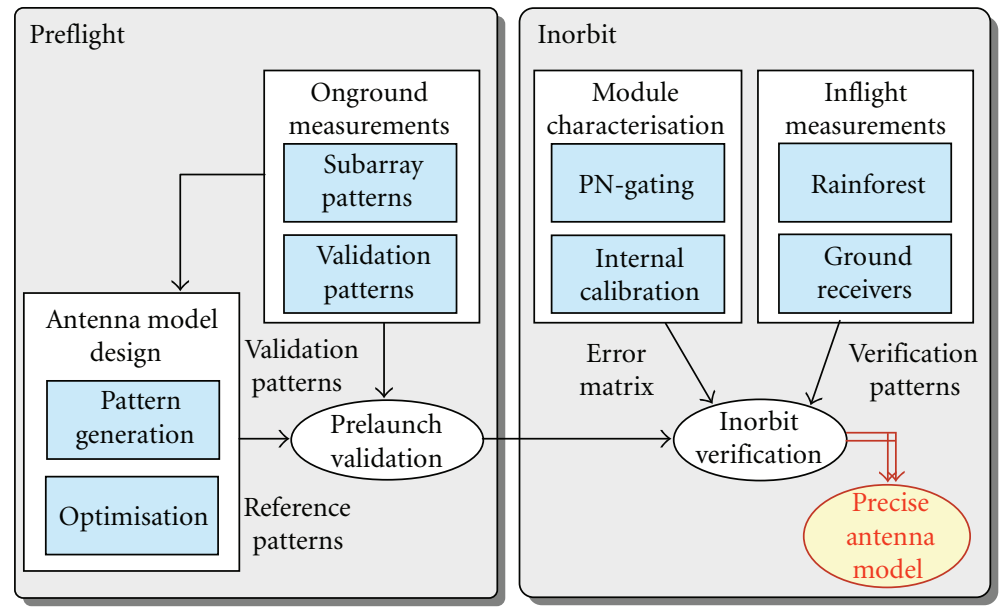

FIgURE 2: Antenna model verification approach.

Both, the excitation laws and the error matrix are different for transmit and receive. In case of the excitation coefficients, the differentiation allows greater flexibility for beam steering. On the side of the error matrix it is mandatory as the paths through the TRM are different, for transmit via the high power amplifier and in receive through the low noise amplifier.

For the TerraSAR-X SAR applications, cuts of the pattern in elevation (at $\alpha=0 \mathrm{deg}$ ) and azimuth $(\varepsilon=0 \mathrm{deg})$ are derived. These are saved into interface tables to be applied for image correction in the SAR processor.

The accuracy of the antenna model is on the one hand mainly determined by the accurately measured embedded subarray patterns and on the other hand by the stability of the instrument. This includes the accurate measurement of the error matrix via PN-Gating as well as the correction of internal variations by the Internal Calibration. To prove the accuracy, sensitive inorbit measurement methods are required as described below.

\section{Prelaunch Validation}

Knowing the design of the Antenna Model, at first an onground validation has been performed. The onground characterisation was realised in two stages. In a first step, the antenna was accurately measured in the Planar Near Field Scanner at and from Astrium GmbH, Germany. In the second step, the correct application of the conventions and input parameters was verified.

For the first step, two kinds of antenna patterns were determined in the Planar Near Field Scanner:

The embedded subarray patterns are required as a direct input into the Antenna Model. For comparison, the patterns of one complete panels or leafs (one third of the whole antenna) was measured.

Due to the dimensions of the whole antenna $(4.8 \mathrm{~m} \times$ $0.7 \mathrm{~m}$ ) and to enable the use of the available measurement chamber, the prelaunch validation was performed only up to the stage of one leaf, for example, one third of the antenna or four panels in a column. To ensure a valid model even for the complete antenna, the accuracy of the antenna model was tracked through the whole built process of the antenna from individual subarray patterns over the patterns of one panel up to the patterns of the three leaves.

Of course, the measurement in an anechoic chamber is not exactly the same as if the antenna is mounted on the satellite. However, as there are no parts of the satellite structure rising into the near field of the SAR Antenna, the effects of the satellite and its mounting structure were expected to be small. On the other hand, the measurements on satellite level in orbit were expected to be better than the leaf-level measurements as three times more TRMs on the complete antenna provide better statistical distribution for the variation of the TRMs.

For the prelaunch validation, all patterns were measured for both polarisation and at five frequencies. After the measurement, the obtained near field results were transformed into the far field using a Fourier transform algorithm. Then, the antenna patterns generated with the Antenna Model using the embedded patterns were compared to the measured patterns of the complete panel and leaf, respectively. The results of this validation on leaf level exemplary for the centre leaf are depicted in Table 1 . It can be seen that the deviation fulfils the required limit of $\pm 0.2 \mathrm{~dB}$ for all beams [8].

After the successful onground validation of the Antenna Model, also the correct application of the conventions in the antenna control unit was verified on satellite level. Here the complete antenna was mounted and connected to the satellite system. Especially the correct handling of the underlying inputs like antenna excitation coefficients, the correct numbering of the antenna elements and the correct steering angle application were successfully tested. Also, the correct update of all changeable antenna parameters like TRM enabling/disabling or update of the excitation coefficients table was verified.

The preflight validation proved a very stable and accurate instrument, which now had to be verified in orbit. 
TABLE 1: The results of the prelaunch validation in elevation.

\begin{tabular}{lcc}
\hline Beam & HH & VV \\
\hline strip_003 & $+0.05 \mathrm{~dB}$ & $+0.15 \mathrm{~dB}$ \\
strip_004 & $+0.10 \mathrm{~dB}$ & $+0.08 \mathrm{~dB}$ \\
strip_005 & $+0.03 \mathrm{~dB}$ & $+0.14 \mathrm{~dB}$ \\
strip_006 & $+0.08 \mathrm{~dB}$ & $+0.08 \mathrm{~dB}$ \\
strip_007 & $+0.13 \mathrm{~dB}$ & $+0.04 \mathrm{~dB}$ \\
strip_008 & $-0.06 \mathrm{~dB}$ & $-0.15 \mathrm{~dB}$ \\
strip_009 & $-0.12 \mathrm{~dB}$ & $+0.10 \mathrm{~dB}$ \\
strip_010 & $+0.10 \mathrm{~dB}$ & $+0.17 \mathrm{~dB}$ \\
strip_011 & $+0.08 \mathrm{~dB}$ & $+0.09 \mathrm{~dB}$ \\
strip_012 & $+0.09 \mathrm{~dB}$ & $+0.05 \mathrm{~dB}$ \\
strip_013 & $+0.02 \mathrm{~dB}$ & $+0.07 \mathrm{~dB}$ \\
strip_014 & $+0.11 \mathrm{~dB}$ & $+0.08 \mathrm{~dB}$ \\
\hline
\end{tabular}

\section{Inorbit Verification}

The inorbit verification of the Antenna Model was performed in the commissioning phase during the first six month after launch. In contrast to the onground measurements, also the influence of the complete satellite structure is covered for azimuth and elevation patterns.

6.1. Antenna Model Verification in Elevation. To verify the Antenna Model in elevation, the simulated relative antenna patterns are compared with measured antenna patterns. The estimates are measured from SAR images acquired over rainforest in the Amazon basin, Brazil. The Amazon rainforest is a homogeneous scatterer [9] (see Figure 3(a)) and the pattern shape is clearly visible in the uncorrected SAR image data (compare Figure 1).

In the processing chain, the accurate position and geometry of the acquisition are determined and annotated after azimuth and range compressions. Then the antenna patterns mapped in the image data are corrected with the available modelled reference antenna patterns [10].

Thus, for pattern estimation, this pattern correction has to be reversed with the used reference patterns to obtain the original impact of the antenna characteristics on the image. Then, the image is freed from disturbances like rivers using an automatic masking algorithm. The radar image is denoted in beta nought $\beta_{0}$ where the backscatter depends on the incidence angle $\theta$. For the comparison however, the gamma nought $\gamma_{0}$ has to be derived via sigma nought $\sigma_{0}$ using the formula [11]:

$$
\gamma^{0}=\frac{\sigma^{0}}{\cos (\theta)}=\beta^{0} \cdot \tan (\theta) .
$$

Finally all azimuth lines are summed up and each pixel position is transformed as function of elevation angle resulting in a so-called Gamma Profile which is a vector of the mean antenna pattern over elevation angle.

For the Antenna Model verification, nominal Stripmap and ScanSAR acquisition were evaluated. The acquisitions had a bandwidth of $150 \mathrm{MHz}$ and a duty cycle of $18 \%$.
TABLE 2: Results of the elevation antenna model verification.

\begin{tabular}{lcc}
\hline Beam & Polarisation & Max. deviation \\
\hline strip_002 & HH & $+0.17 \mathrm{~dB}$ \\
strip_002 & VV & $-0.19 \mathrm{~dB}$ \\
strip_002 & HV & $+0.10 \mathrm{~dB}$ \\
strip_002 & VH & $-0.18 \mathrm{~dB}$ \\
strip_007 & HH & $+0.10 \mathrm{~dB}$ \\
strip_007 & VV & $+0.17 \mathrm{~dB}$ \\
strip_007 & HV & $+0.13 \mathrm{~dB}$ \\
strip_007 & VH & $+0.19 \mathrm{~dB}$ \\
strip_013 & HH & $+0.08 \mathrm{~dB}$ \\
strip_013 & VV & $+0.17 \mathrm{~dB}$ \\
\hline
\end{tabular}

As said before, to minimize the inorbit calibration effort, only few beams were selected for verification. These beams were in a further step also used for absolute calibration, that is, for the determination and verification of the absolute calibration factor.

An exemplary result of the Antenna Model in Elevation verification is depicted in Figure 3(b). The noisy ripple (in green) is the gamma profile, which now can be compared to the modelled reference pattern depicted in red.

The results show an excellent accordance between the simulated antenna pattern and the measured gamma profiles. This can be seen in Figure 3(c), where the deviation between the reference antenna pattern and the estimated pattern is depicted for the exemplary beam. Additionally, by fitting a blue curve into the profile, a noise-free picture is obtained.

Table 2 summarizes the measurements of the selected beams as well as the maximal deviation between measurement and reference patterns. Several other beams have been measured as well, showing similar performance. Hence, the deviation and consequently the accuracy of the Antenna Model are within $\pm 0.2 \mathrm{~dB}$ (peak-to-peak) for the pattern shape.

6.2. Antenna Model Verification in Azimuth. In the next step, the antenna model has to be verified also in flight direction. For this purpose, using ground receivers, the antenna can be determined during an overflight. As only the transmitpatterns can be measured with the ground receivers, the accuracy requirement with $\pm 0.1 \mathrm{~dB}$ (peak-to-peak) is half the one as for the two-way patterns. With ground receivers it is furthermore possible to verify the side-lobes being not possible over rainforest.

The verification of the antenna pattern in azimuth direction was performed for transmit pattern using the DLR ground receivers [12]. These receivers record the amplitude of the pulses transmitted by the SAR antenna as function of time and due to the flight movement, a cut through the antenna pattern is recorded. Transformed to the antenna azimuth angles and corrected by position information, the azimuth antenna pattern is obtained and can be compared to the modelled pattern. The measurements were performed for patterns over the whole specified angular range of the SAR 


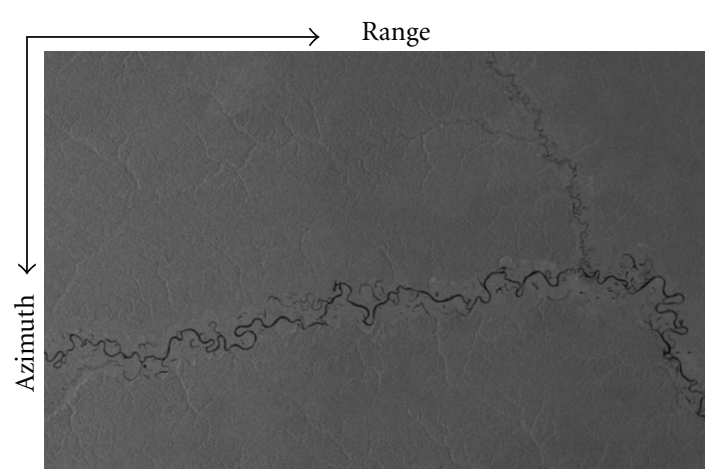

(a)

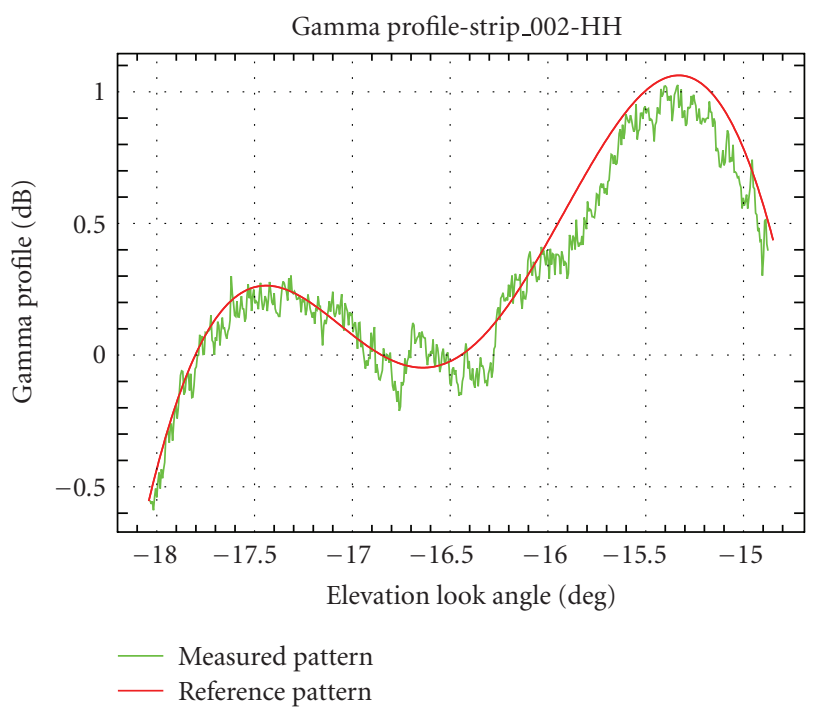

(b)

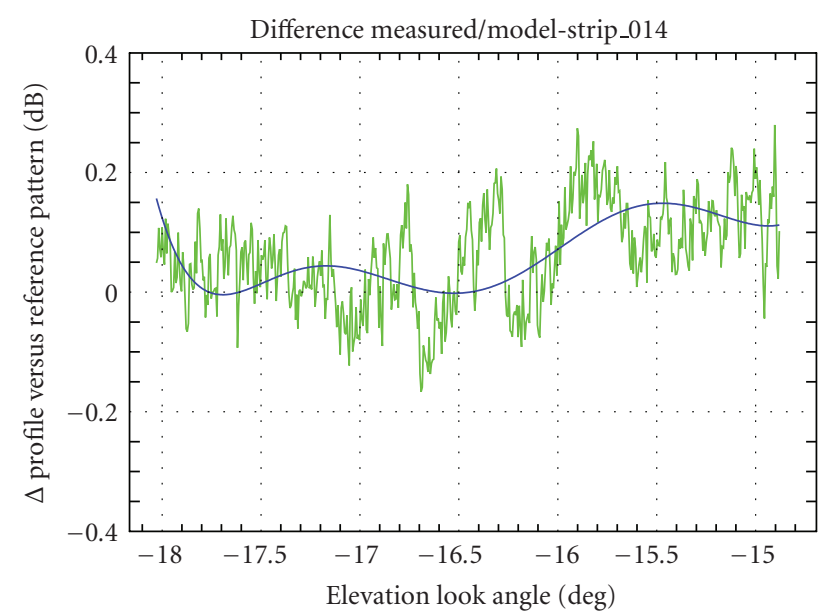

(c)

Figure 3: (a) Amazon rain forest scene used for antenna model verification. (b) Gamma profile of the antenna pattern extracted from rainforest (green curve) and reference pattern (red line) for comparison. (c) Deviation between gamma profile and reference pattern (green curve) and fit through the deviation (blue line).

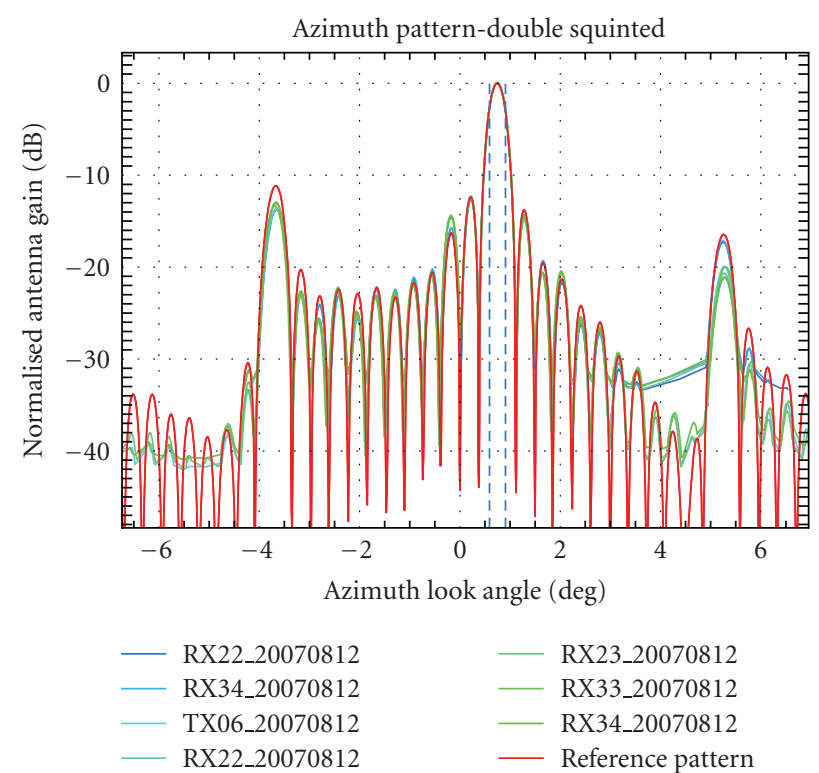

(a)
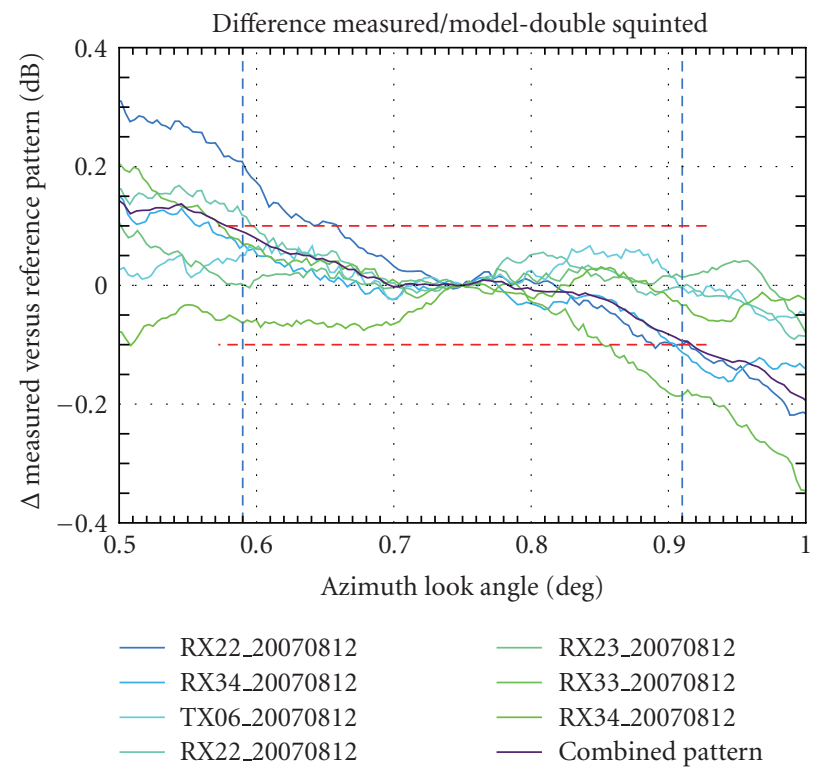

(b)

FIgURE 4: (a) Transmit patterns compared to a reference pattern, blue to green: measured profiles, red: reference patterns derived by the antenna model. (b) Deviation between measured and reference pattern.

antenna, that is, for low, mid and high incidence angles. Furthermore ground receivers were placed across each swath being measured at near, mid and far range.

Figure 4(a) shows the special case of a double squinted beam nominally only needed for high resolution spotlight acquisitions. In this case a beam steered to angles of $+0.75^{\circ}$ in azimuth and $-16.5^{\circ}$ in elevation which is at the specified limits for antenna steering. This beam was specially commanded to be fixed, as in nominal spotlight mode the beam would switch up to 123 times during one acquisition. 
TABLE 3: Results of the azimuth antenna model verification.

\begin{tabular}{lcc}
\hline Beam & Transmit polarisation & Max. deviation \\
\hline strip_002 & $\mathrm{H}$ & $+0.09 \mathrm{~dB}$ \\
strip_002 & $\mathrm{V}$ & $+0.09 \mathrm{~dB}$ \\
strip_007 & $\mathrm{H}$ & $-0.08 \mathrm{~dB}$ \\
strip_007 & $\mathrm{V}$ & $-0.07 \mathrm{~dB}$ \\
strip_013 & $\mathrm{H}$ & $-0.07 \mathrm{~dB}$ \\
strip_013 & $\mathrm{V}$ & $-0.08 \mathrm{~dB}$ \\
\hline
\end{tabular}

The antenna patterns measured by ground receivers during one pass are depicted in green to blue and the corresponding reference pattern derived by the model is depicted in red. The resulting deviation between these measurements and the reference pattern are shown in Figure 4(b) by the purple line, whereby all measurements derived from the ground receiver deployed during one pass were averaged in order to reduce the error contribution of the ground receiver.

Table 3 summarizes the results for the selected beams of the azimuth verification. As for elevation, the Antenna Model verification shows extraordinary results, that is, the resulting deviation within the main beam and consequently the accuracy of the model is within the required $\pm 0.1 \mathrm{~dB}$.

6.3. Verification of the Beam-to-Beam Gain Prediction. Besides the verification of the pattern shape, the capability of the Antenna Model to predict the gain offset between different beams is of great importance. First, this is required for the ScanSAR processing, where four different beams with different antenna gains are acquired, corrected with their corresponding pattern and combined into one image. Second, with an appropriate gain prediction, only one absolute calibration factor can be derived for the complete system and not all beams have to be measured during the absolute calibration [5].

The beam-to-beam gain prediction is verified evaluating ScanSAR data. In ScanSAR operation, the beam is switched sequentially from burst to burst between a set of four neighbouring swaths to get a broader swath width than for normal Stripmap acquisitions. By generating the unnormalised gamma profile for each of the four swaths, the relative gain deviation can be determined.

In order to obtain the beam-to-beam gain prediction over a wide range of elevation angle including the full performance range (between $-15 \mathrm{deg}$ and $+9 \mathrm{deg}$ antenna look angle), different sets of ScanSAR acquisitions were combined. The result is shown in Figure 5(a). Indeed, the images were acquired over different parts of the rainforest caused by switching the beam in ScanSAR operation. Thus, each illuminated part of the rainforest has different vegetation and consequently different backscatter. Consequently, each complete set of four beams was connected to its predecessor set within the overlapping region.

Figure 5(b) shows the deviation between the profiles measured and the corresponding reference pattern. Figure 5(c) emphases only the deviation between the overlapping regions of two neighbouring swaths. However, the

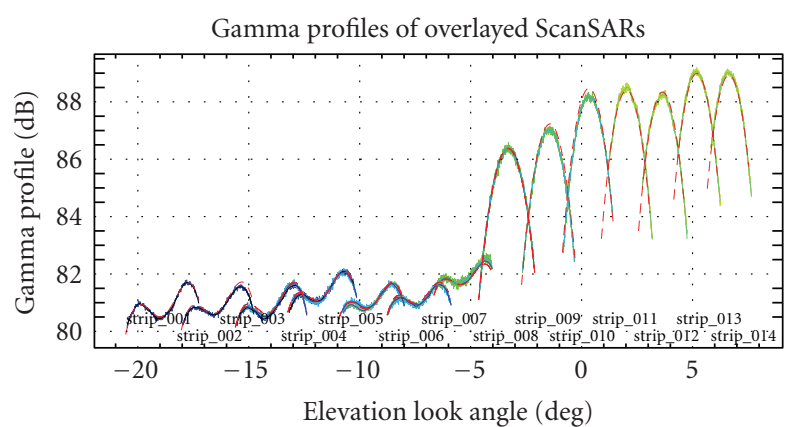

(a)

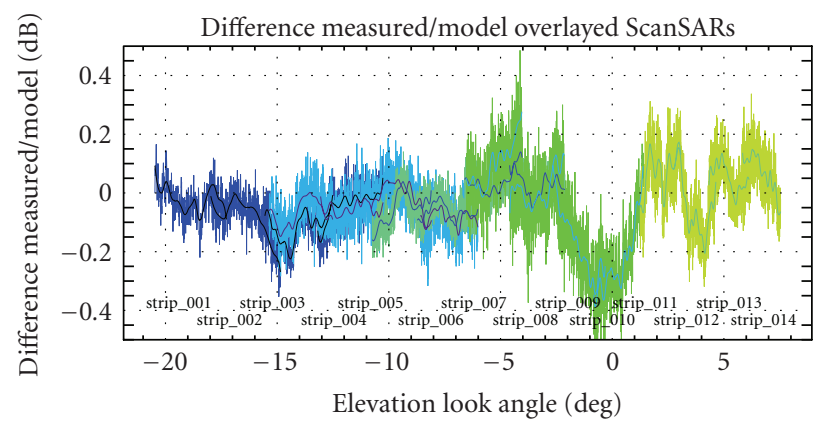

(b)

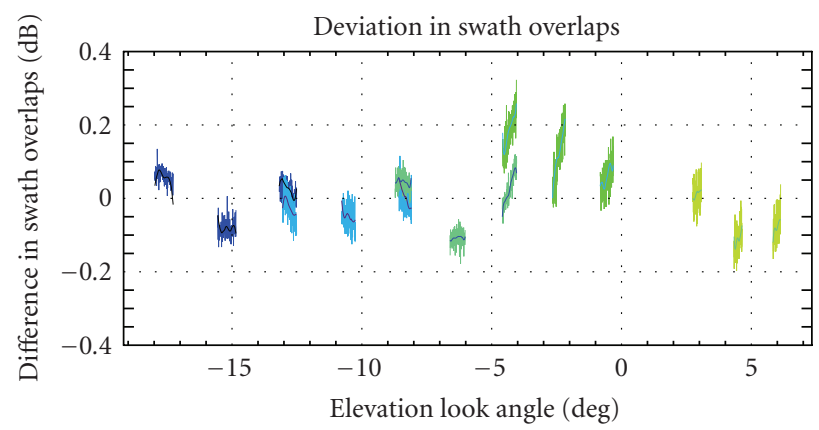

(c)

FIGURE 5: (a) Verification of the beam-to-beam gain prediction using ScanSAR images, blue to green: measured gamma profiles, red: reference patterns derived by the antenna model. (b) Deviation between gamma profile and the reference patterns remaining within the range of $\pm 0.2 \mathrm{~dB}$. (c) Deviation within the overlapping areas of Figure 5(b).

results again have an excellent accuracy of below $\pm 0.2 \mathrm{~dB}$ peak-to-peak over the whole angular range and no drift is visible. The slight deviation at about 0 deg elevation angle in Figure 5(b) results from bad weather conditions disturbing the acquisition. Because illuminating an area of about $560000 \mathrm{~km}^{2}$ across the rainforest, nearly twice the area of Germany, it is natural to find individual regions with heavy rainfall rates, especially across the rainforest.

A second method to verify the beam-to-beam gain prediction was performed by deploying ground receivers in the overlapping regions of two neighbouring swaths and recording the transmit azimuth patterns. For this purpose 


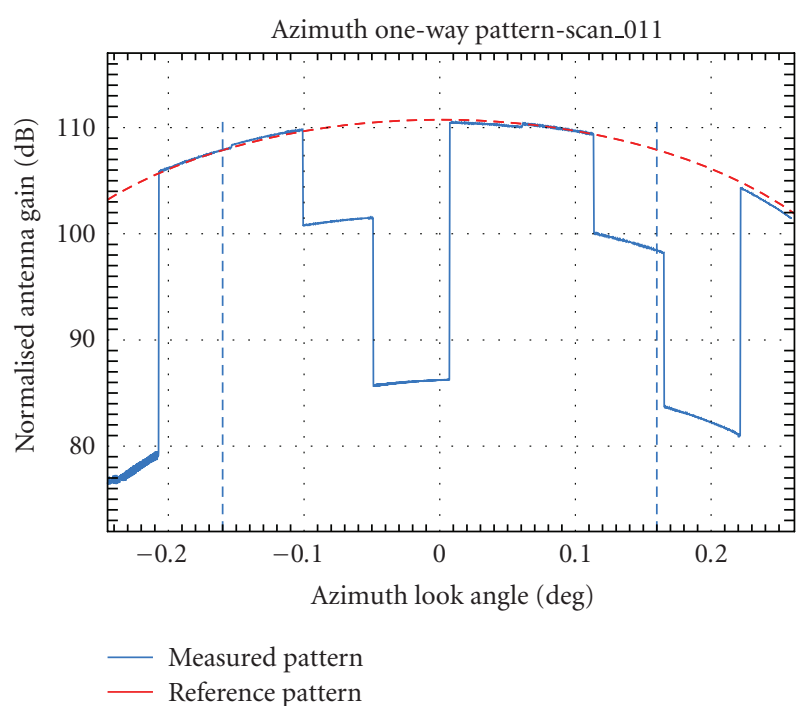

(a)

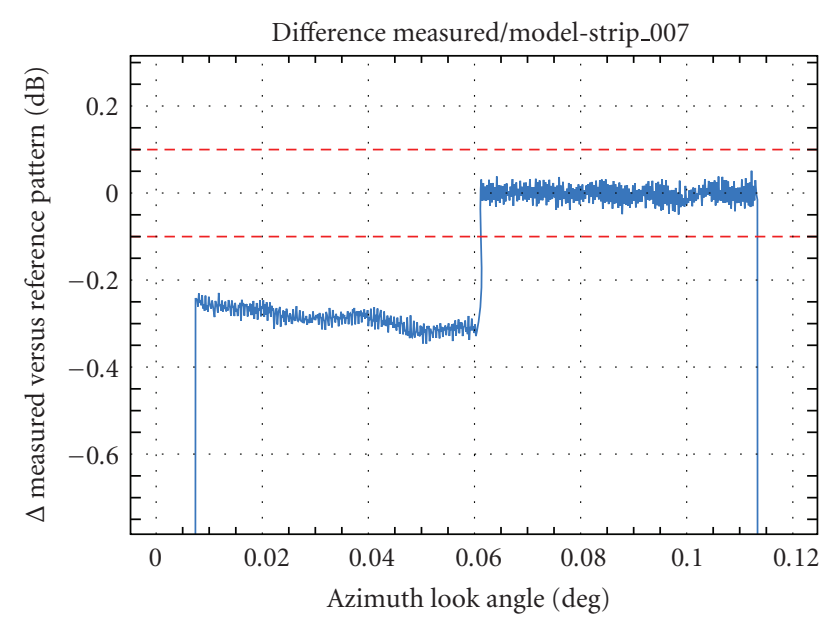

(b)

Figure 6: (a) Verification of the beam-to-beam-gain prediction using ground receivers. (b) Zoom into switching region between two swaths.

the instrument was operated again in ScanSAR mode and as shown in Figure 6(a) the switching of the instrument between the four beams during one pass is clearly visible by the received pulses. The deviation between the overlapping beams can be likewise compared with the Antenna Model. And even here, that is, measuring one-way patterns by ground receivers and the interrelated demand on higher accuracy (see above), the maximum deviation is within the required $\pm 0.1 \mathrm{~dB}$ peak-peak.

With the properly working Antenna Model, the absolute calibration factor required for deriving the radar backscatter coefficient of any target within an image, does not have to be measured for all relevant beams. It is measured only for one beam and verified for the same representative beams as used for the antenna model. By this approach it is possible to shorten the time and the effort extremely for the absolute radiometric calibration performed during the commissioning phase of an SAR system. In case of TerraSAR$\mathrm{X}$ with the verified Antenna Model, it was sufficient to measure only 3 of the 12000 different beams: one with low, one with mid and one with high incidence angle. The measurement results show an absolute radiometric accuracy of $0.31 \mathrm{~dB}$ (1-sigma) [5].

\section{Conclusion}

The TerraSAR-X Antenna Model is utilised for generating the reference antenna patterns for processing and for beam optimisation. A high accuracy of the Antenna Model is achieved by accurately onground measured embedded subarray patterns as well as a highly accurate internal calibration of the instrument. To ensure the specified product quality, its accuracy has been verified in orbit during the commissioning phase following the TerraSAR-X launch in June 2007. The results show the excellent accuracy of both, the whole TerraSAR-X system itself and the Antenna Model. The Antenna Model was verified on providing the reference antenna patterns with an accuracy of better than $\pm 0.2 \mathrm{~dB}$ (peak-to-peak) for image correction in elevation using Amazon rainforest. In azimuth, where transmit patterns were determined with ground receivers, an accuracy of $\pm 0.1 \mathrm{~dB}$ (peak-to-peak) was achieved. The prediction of the beamto-beam gain offset could be verified with $\pm 0.2 \mathrm{~dB}$ (peakto-peak) accuracy enabling accurate image referencing and a short commissioning phase.

The excellent results of TerraSAR-X show that the Antenna Model approach was successfully applied and the approach should be used for future system.

\section{Acknowledgments}

The authors would like to thank the whole TerraSAR-X team and especially to the HR calibration team for supporting the Antenna Model verification with an uncountable number of calibration campaigns and their unweary effort at every possible weather and environmental conditions. Furthermore a special thank to the Astrium GmbH for performing the highly accurate embedded antenna pattern measurements and onground validation and developing the TerraSAR-X as such an highly accurate SAR system.

\section{References}

[1] M. Zink and B. Rosich, "Antenna elevation pattern estimation from rain forest acquisitions," in Proceedings of the Envisat Calibration Review (ECR '02), European Space Agency, Noordwijk, The Netherlands, September 2002.

[2] S. Buckreuss, R. Werninghaus, and W. Pitz, "The German satellite mission TerraSAR-X," in Proceedings of IEEE International Radar Conference (RADAR '08), pp. 1-5, Rome, Italy, May 2008.

[3] B. Grafmüller, A. Herschlein, and C. Fischer, "The TerraSAR$\mathrm{X}$ antenna system," in Proceedings of IEEE International Radar Conference (RADAR '05), pp. 222-225, Washington, DC, USA, May 2005. 
[4] M. Schwerdt, D. Hounam, J. L. Álvarez-Pèrez, and T. Molkenthin, "The calibration concept of TerraSAR-X: a multiplemode, high-resolution SAR," Canadian Journal of Remote Sensing, vol. 31, no. 1, pp. 30-36, 2005.

[5] M. Schwerdt, B. Bräutigam, M. Bachmann, B. Döring, D. Schrank, and J. H. Gonzalez, "Final results of the efficient TerraSAR-X calibration method," in Proceedings of IEEE Radar Conference (RADAR '08), pp. 1-6, Rome, Italy, May 2008.

[6] B. Bräutigam, M. Schwerdt, M. Bachmann, and M. Stangl, "Individual T/R module characterisation of the TerraSAR-X active phased array antenna by calibration pulse sequences with orthogonal codes," in Proceedings of the 27th IEEE International Geoscience and Remote Sensing Symposium (IGARSS '07), pp. 5202-5205, Barcelona, Spain, June 2007.

[7] C. A. Balanis, Antenna Theory: Analysis and Design, John Wiley \& Sons, New York, NY, USA, 2nd edition, 1997.

[8] M. Bachmann, M. Schwerdt, B. Bräutigam, B. Grafmüller, A. Herschlein, and J. L. Álvarez-Pérez, "The TerraSAR-X antenna model approach," in Proceedings of the 2nd International ITG Conference on Antennas (INICA '07), pp. 139-142, Munich, Germany, March 2007.

[9] A. P. Luscombe and A. Thompson, "RADARSAT-2 calibration: proposed targets and techniques," in Proceedings of IEEE International Geoscience and Remote Sensing Symposium (IGARSS '01), vol. 1, pp. 496-498, Sydney, Australia, July 2001.

[10] J. L. Álvarez-Pèrez, M. Schwerdt, and M. Bachmann, "TerraSAR-X antenna pattern estimation by a complex treatment of rain forest measurements," in Proceedings of IEEE International Geoscience and Remote Sensing Symposium (IGARSS '06), pp. 3857-3860, Denver, Colo, USA, July-August 2006.

[11] J. E. Laycock and H. Laur, "ERS-1 SAR antenna pattern estimation," ES-TN-DPE-OM-JL01, iss. 1, rev. 1, September 1994, http://earth.esa.int/ers/instruments/sar/sar_AP_est.

[12] B. Döring, M. Schwerdt, and R. Bauer, "TerraSAR-X calibration ground equipment," in Proceedings of the Wave Propagation in Communication, Microwave Systems and Navigation (WFMN'07), pp. 91-96, Chemnitz, Germany, July 2007. 

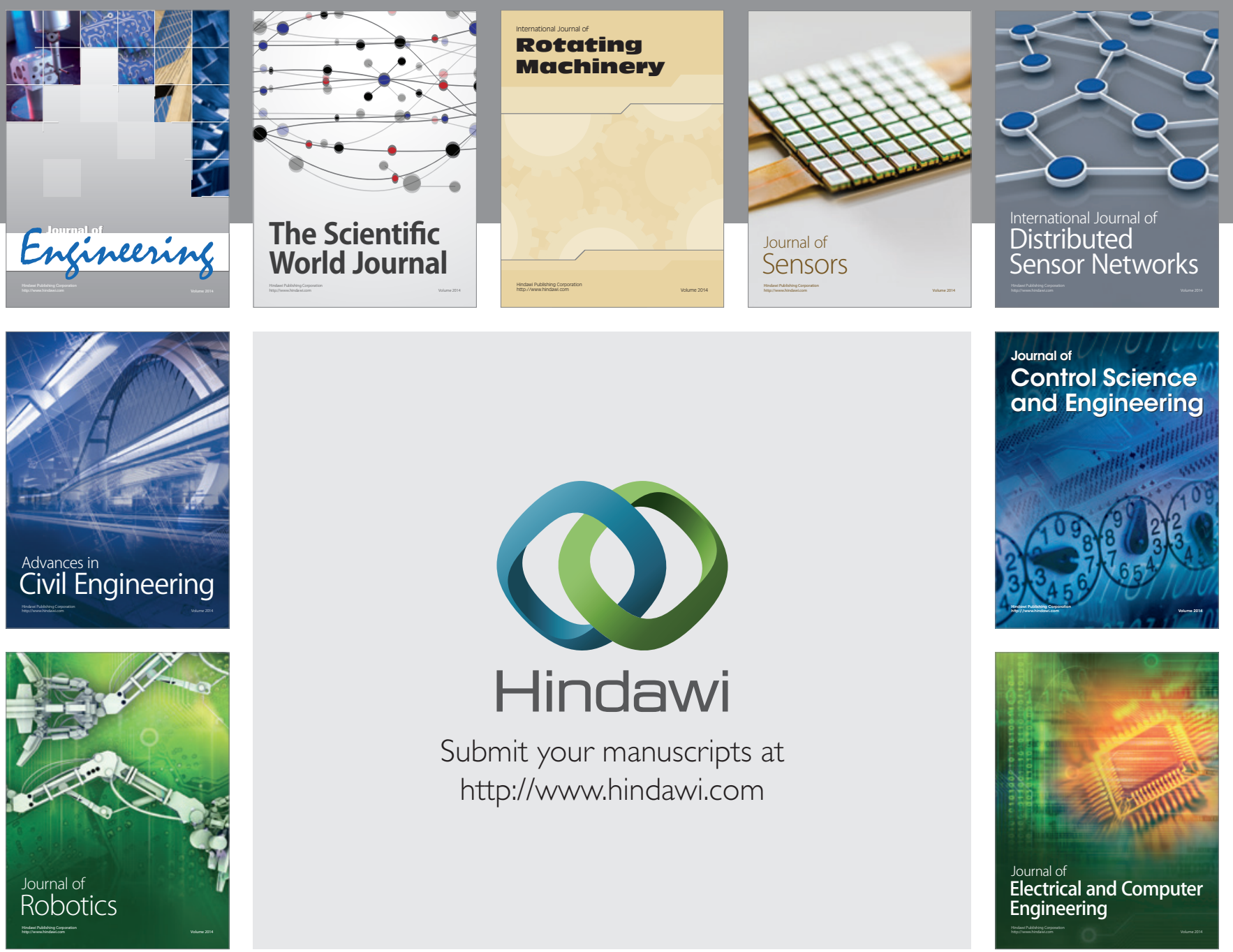

Submit your manuscripts at

http://www.hindawi.com
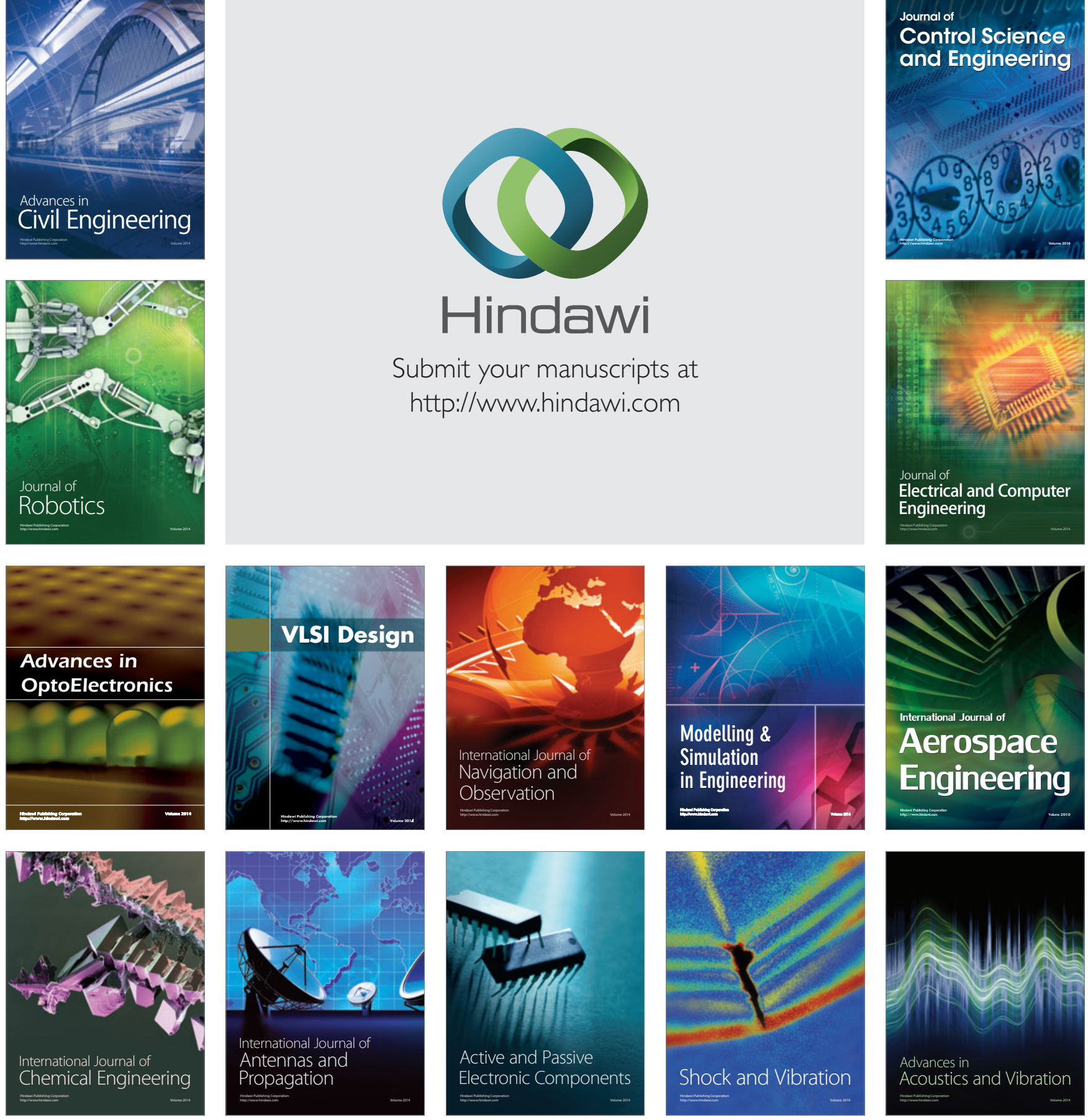